\title{
Making Prescribing Safer in General Practice: Need for Medical Record Keeping in Pakistan
}

Jameel ur Rahman

\begin{abstract}
Prescribing medicines without knowing patient's chronic illnesses, current medications, drug allergies is risky. It may lead to enormous patient suffering in the form of iatrogenic health damage and monetary burden, as in our country Pakistan; it is the patients who pay the costs. The main cause is un-awareness of the concept of maintenance of patients' medical records in out of hospitals healthcare providers. In this article the effects of this continuing dangerous state is discussed and very basic way to maintain medical record, pertinent to our socio-economic conditions is presented which can lead to safe prescribing. It can lay ground for eventual quality record keeping in our country. These suggestions are very basic, on "something is better than nothing" principle. They in no way are a substitute to quality record keeping. The only aim is; safe prescriptions.
\end{abstract}

KEYWORDS: Safe clinical practice, Safe prescribing, Patient records, Medical records.

This article may be cited as: Rahman J. Making Prescribing Safer in General Practice: Need for Medical Record Keeping in Pakistan. J Liaquat Uni Med Health Sci. 2017;16(01):68-71. doi: 10.22442/jlumhs. 171610509

\section{INTRODUCTION}

To prescribe a medicine when it is contraindicated in that patient is potentially dangerous with even fatal outcomes. It is imperative to know the allergies, concomitant illnesses and current medications in safe clinical practice.

How can that be done? The answer is medical record keeping. Good record keeping is a costly affair, it needs whole lot of infrastructure with Information Technology personnel and expensive gadgets and computers and persistent supply of electricity. It is an advanced field by itself.

This article is for a resource poor country like ours, where we even do not think of patient record keeping due to costs and infrastructure involved. Erratic supply of electricity is another perpetual issue. This presentation is about how to implement a system which, though not ideal, but will atleast solve the purpose substantially in our country. The goal is only one; safe prescription of medicines. Let us not as physicians add to our patient's sufferings by our prescriptions, by unknowingly inflicting more harm than good.

\section{Medical record keeping in Pakistan}

There is absent to minimal medical record keeping in clinical practice, mainly primary care setting in our country. Even there is no record for future referral by the attending physician himself, except for may be, just registration of patient's name. It is also poor in some of the tertiary care centers in big cities of Pakistan ${ }^{1}$. It has been pointed out earlier as well while conducting studies on tuberculosis ${ }^{2}$, maternal health ${ }^{3}$, and breast cancer ${ }^{4}$ by various research groups. Even if a file is made in a hospital, that is not retrievable at the subsequent visit by the patient. It just adds to already existing unmarked piles of files in the store, hence making it functionally absent record keeping. The reason seems to be the lack of awareness of the need of record keeping.

\section{Advantages of medical record keeping}

1. Demographic record: Changing weight of a patient is an important clue. Weight loss may be a marker of many illnesses. From Diabetes mellitus to more sinister ones like neoplastic disorders. We may only pick this important piece of information, if we know the earlier weights from his records.

2. Record of drug allergies: Drug allergies (IgE mediated reactions) of all grades of severity to unsuspecting medications e.g: analgesics, antibiotics are quite common. Similarly drug intolerances ( non IgE mediated reactions) too are common.

3. Record of vital signs: Raised blood pressure at current presentation may be inconclusive, but if found higher at earlier visits may confirm a diagnosis of hypertension. Similarly persistent documented low grade fever will point to an array of differential diagnosis.

4. Composite diagnoses: A chronically jaundiced person due to Gilberts Syndrome need not be made to pay for unnecessary liver function tests by every new physician, if the diagnosis is known to him. Another patient may be on oral anticoagulants, requiring specific precautions. A G6PD 
deficient patient needs to avoid some medicines to avoid hemolysis. Someone with peptic ulcer disease or chronic renal failure will have to avoid NSAIDs, etc. (As in image 2, NSAIDs are marked to be avoided since the patient has diabetic nephropathy) and all this is possible only if a medical record is available.

5. Record of laboratory workup: Baseline Hemoglobin values, high serum creatinine, LFTs and serum electrolytes abnormalities, etc can guide choice of medicines to avoid further organ damage. Moreover it avoids unnecessary repetition of lab tests thus cutting down patient's financial burden.

6. Radiological and electrocardiographic (ECG) record: Baseline cardiothoracic ratio, pulmonary parenchymal shadows and ECGs may be followed up for comparison. Unnecessary repeat radiographs may be avoided.

7. Current medications: Some complaints are due to side effects of medications. Adverse drug reactions (ADR) constitute significant proportion of patient suffering. In a study $3 \%$ of all deaths in general population were due to $A D R^{5}$. In USA it is amongst 5 leading causes of death ${ }^{6}$. Mere stopping the culprit medicine is all that is required provided that is known. It is only possible by record keeping. Moreover it may lead us to the diagnosis if the presenting complaint is due to side effect of some medicine. For instance tachycardia and or hand tremors due to Beta agonists, bradycardia due to beta blockers, diarrhea due to Metformin, constipation due to iron supplements, raised ALT and AST due to Co- Amoxiclav etc. A physician will not suggest phosphodiesterase inhibitors e.g: sildenafil (Viagra) if he knows the patient takes nitrates Or he will avoid omeprazole if his patient is taking clopidogrel (Plavix). Patients on chronic maintenance steroids develop acute adrenal crisis; if steroid dose is not doubled in even apparently minor febrile illnesses.

8. Growth charts: It is an essential part of the medical record of a child, as is the immunization record.

9. Continuity of care: Wherever the patient may go, be it another city, the new physician may find all the relevant record with the patient.

10. Others: Medico-legal cover and research are other uses of strictly maintained quality records.

\section{How it can be done?}

The following plan is on the model of individuals personal record keeping diaries maintained in the West ${ }^{7}$.

a) The patient will purchase one time two ring file costing around 100 rupees. This becomes his "Personal Medical File" (Image I). The patient would always take his file to any physician whenever needed.

b) The first attending physician would enter the patient's particulars on a standardized proforma (Image II).

c) From second page, prescriptions to be clipped. Every prescription should however be dated and should contain pulse, blood pressure, temperature, weight at every visit. It should mention major presenting complaints, relevant clinical findings, suggested labs if any and the provisional diagnosis (Image III). These vital signs become the basis and source of diagnosing diseases in future as mentioned above.

d) While writing a prescription, he can place a carbon copy underneath to get 2 copies. One for the patient's medical file and other for his own record as below.

e) Investigations results to be attached at the end of the file.

f) This file will be handed over to the patient at the conclusion of the consultation.

g) The patients will have to keep their personal medical file at a handy place at home and will carry to any doctor they visit later.

h) On the visit to the physician, the physician should ask for his file, go through it to see relevant details for possible clues to the diagnosis and safe prescription then start from step $C$ above.

Legislation and directions can make it mandatory for all points of patient care to have appropriate workable medical record system. The room or file racks should be in lock since medical files are personal documents of every patient.

In this way, by little additional effort by the physician patients medical records will automatically be maintained. This simple step is what will be invaluable in providing essential background information for safe prescribing by the next physician. It will have immense effect in rationalizing overall clinical practice.

\section{IMAGE I: HARD COVER 2 CLIP FILE}

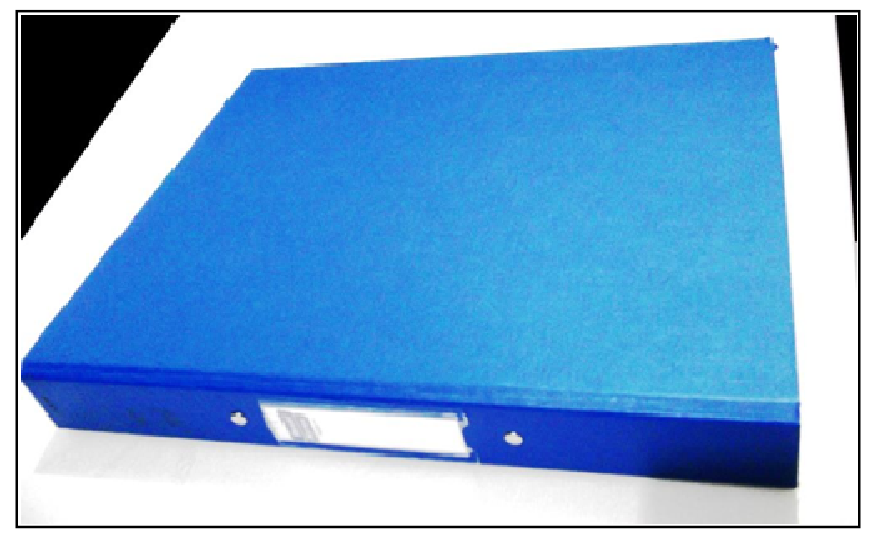




\section{IMAGE II: FIRST PAGE WITH DEMOGRAPHIC DE- TAILS OF THE PATIENT. IT MAY HAVE MENTION OF DIAGNOSES AND CHRONIC MEDICATIONS}

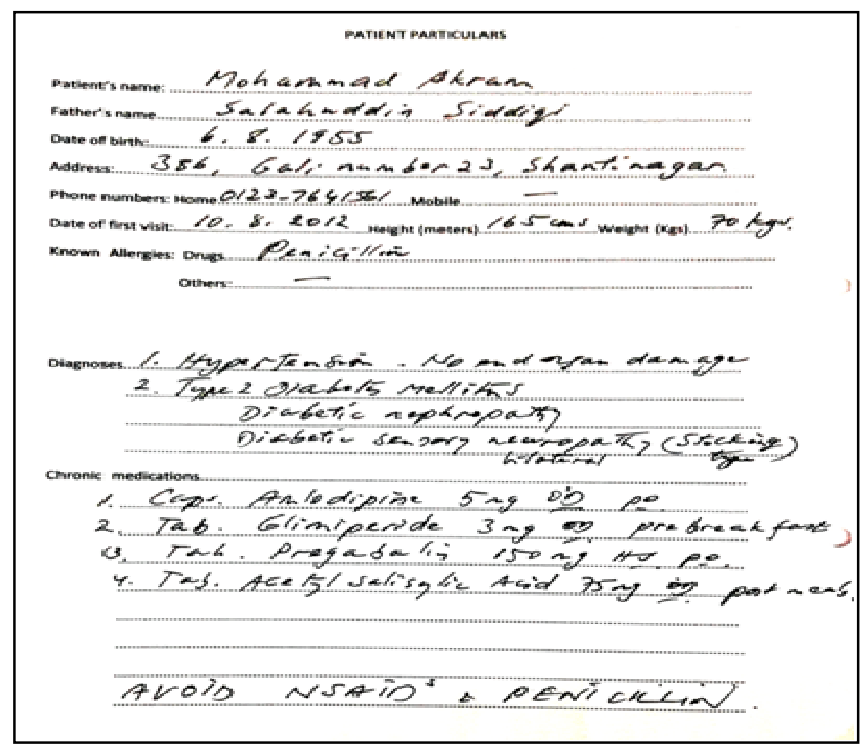

IMAGE II: EVERY ATTENDING PHYSICIAN TO ATTACH A COPY OF HIS PRESCRIPTION IN THE FILE

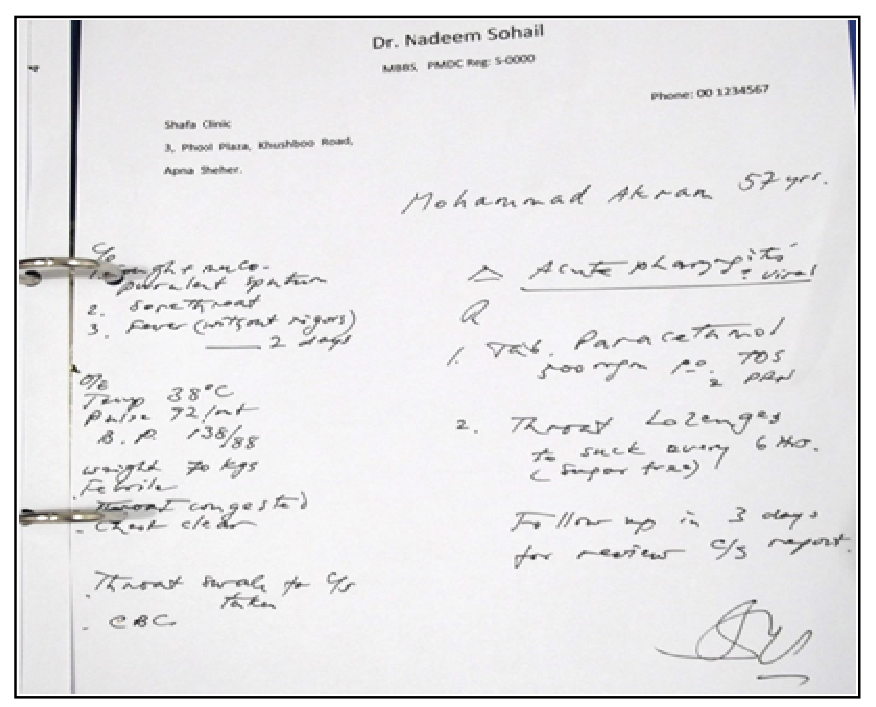

Role of healthcare regulating bodies

Ministry of Health and Pakistan Medical and Dental Council both have been striving hard to improve healthcare in our country. They should step forward in this aspect of patient care as well. They can highlight the importance of the medical record keeping and ensure its implementation.

\section{DISCUSSION}

Simplest working definition of medical record keeping can be; "Any appropriate recording and retrieval mechanism at any point of patient care by which the prescribing physician, can know his patients medical details". It is to ensure safe prescriptions and to avoid duplication, interaction and contraindications of medications.

- For instance: Prescribing an NSAID or a PPI, when he is already taking one, from another physician. (Duplication)

- Prescribing Omeprazole when he is taking Clopidogrel (Interaction)

- Ordering an Intramuscular injection in someone who is already on anticoagulant; for instance warfarin(Contraindication).

- Prescribing antibiotic in someone who is allergic to it (Hypersensitivity It is negligence, with potential fatal implications).

All above are physician related harms which are totally avoidable. Even in the places with good record keeping like USA, "medication errors cause at least one death every day and harm approximately 1.3 million people annually" ${ }^{8}$. What would be the scale of iatrogenic damage in our country is anyone's imagination.

\section{CONCLUSION}

There have been previous attempts to introduce health information systems in Pakistan, but that has been from the health planner's point of view ${ }^{9}$, hence of no practical use in clinical practice. It is well known that Electronic Medical Records (EMRs) though accurate can be quite difficult to establish and maintain in developing countries ${ }^{10}$. Affordability of technology and uninterrupted power supply are main reasons. In Pakistan we can do something even though very basic. These simple steps are not dependent on technology or on constant supply of electricity. These are just few suggestions which are presented. There can be modifications to more acceptable and practical ways. Patients will readily cooperate if informed about the use and value of their records in safe medications for their own benefit.

Medical records serve many functions but their primary purpose is to support patient care ${ }^{11}$.

Physicians may be reluctant to take responsibility, how so ever small. In fact, they are supposed to do that as part of their professional duty, as laid down by the Pakistan Medical and Dental Council ${ }^{12}$. It will be a great service to the patient and the profession. It will make their practice safer as well.

\section{REFERENCES}

1. Aziz $\mathrm{S}, \mathrm{Rao} M \mathrm{MH}$. Existing record keeping system in government teaching hospitals of Karachi. J Pak Med Assoc 2002; 52(4):163-74.

2. Shabbir I, Iqbal R, Anwar M, Qadeer E, Ahmed N. An improved record system for tracing outcome of "transferred-out" DOTS patients. EMHJ;2011;17 
(2):88-92.

3. Ali M, Kuroiwa C. Accurate record keeping in referral hospitals in Pakistan's North West frontier province and Punjab: a crucial step needed to improve maternal health. J Pak Med Assoc 2007;57(9):443-6.

4. Afsar NA, Kulsoom B, Mateen A, et al. Breast cancer pattern and chemotherapy response: An institutional study in Pakistan. Asia Pacific J Cancer Prev. 2010;11:825-30.

5. Wester K, Jonsson AK, Spigset O, et al. Incidence of fatal adverse drug reactions: a population based study. $\mathrm{Br} J$ Clin Pharmacol 2008; 65(4): 573-9.

6. Lazarou J, Pomeranz BH, Corey PN. Incidence of adverse drug reactions in hospitalized patients: a meta-analysis of prospective studies. JAMA 1998;279(15):1200-5.

7. Medical Record Keeping Diary-Record and track personal and family medical history[Internet]. Available from: URL; http:www.mydiaryforlife.com.
8. US Food and Drug Administration [Internet]. Medication error reports. Silver Spring, MD. USA. Available from: https://www.fda.gov/drugs/ drugsafety/medicationerrors/ucm080629.htm.

9. Ali M, Horikoshi $Y$. Situation analysis of health management information system in Pakistan. Pak J Med Res. 2002;41(2):64-69.

10. Tomasi E, Facchini LA, Maia MF. Health informatics technology in primary health care in developing countries: a literature review. Bull World Health Organ. 2004;82(11):867-74.

11. Mann R, Williams J. Standards in medical record keeping. Clin Med (Lond). 2003;3(4):329-32.

12. PMDC: Code of ethics of practice for medical and dental practitioners 2002; Part IV Ethical standards of professional competence, care and conduct. Paras 25:2(f), 26, 27 and 42. Available from: www.pmdc.org.pk/Ethics/tbid/101/ Default.asp.

AUTHOR AFFILIATION:

Dr. Jameel ur Rahman

Consultant Internal Medicine

Ain Al Khaleej Hospital, Al Ain, United Arab Emirates

Email: JRahman@ak-hospital.com 